Prospective Trial

\title{
Comparative Efficacy of Levobupivacaine and Ropivacaine for Epidural Block in Outpatients with Degenerative Spinal Disease
}

Takashi Egashira, MD1', Makoto Fukusaki, MD¹, Hiroko Araki, MD',

Akiko Sakai, MD', Mai Okada, MD¹, Yoshiaki Terao, MD¹, and Tetsuya Hara, MD²

From: ${ }^{1}$ Department of Anesthesia, Nagasaki Rosai Hopital, Nagasaki, Japan; 2Department of Anesthesiology, Nagasaki University School of Medicine, Nagasaki, Japan

Address Correspondence: Takashi Egashira MD, Department of Anesthesia Nagasaki Rosai

Hopital, 2-12-5, Setogoshi, Sasebo, Nagasaki, 857-0134, Japan E-mail:

tegashira62@yahoo.co.jp

Disclaimer: There was no external funding in the preparation of this manuscript. Conflict of interest: Each author

certifies that he or she, or a member of his or her immediate

family, has no commercial association (i.e., consultancies, stock ownership, equity interest, patent/licensing arrangements, etc.) that might pose a conflict of interest in connection with the

submitted manuscript.

Manuscript received: 03-24-2014

Revised manuscript received: 06-23-2014

Accepted for publication: o6-26-2014

Free full manuscript: www.painphysicianjournal.com
Background: Levobupivacaine has less toxic potential on both the cardiovascular and central nervous system and has been widely used for postoperative epidural analgesia in surgical patients. However, there are few reports on the efficacy of epidural levobupivacaine in outpatients with lumbosacral radiculopathy. This study was carried out to evaluate the comparative efficacy of levobupivacaine and ropivacaine for epidural block in outpatients with degenerative spinal disease and sciatica.

Objective: We studied 32 patients (19 men and 13 women) with degenerative spinal disease and sciatica.

Study Design: The study was performed in a prospective, randomized, double blind, and crossover fashion.

Setting: University medical facility.

Methods: The epidural block was produced with a caudal approach $(0.125 \%$ levobupivacaine or $0.2 \%$ ropivacaine, $15 \mathrm{~mL}$ ). The upper level of analgesia, lumbosacral pain, motor blockade, and hemodynamic changes were evaluated by pin prick, visual analogue scale (VAS), Bromage scale, and arterial blood pressure and heart rate at 15, 30, 60, and 90 minutes after epidural block, respectively. The recovery time to mobilization, ambulation, and spontaneous micturition were measured.

Results: There were no significant differences $(P<0.05)$ in the upper level of analgesia, VAS, and Bromage scale between $0.125 \%$ levobupivacaine and $0.2 \%$ ropivacaine throughout the time course. There were no significant differences in the recovery times to mobilization, ambulation, and spontaneous micturition between $0.125 \%$ levobupivacaine and $0.2 \%$ ropivacaine. There were no significant differences in arterial blood pressure and heart rate between the 2 trials throughout the time course.

Conclusion: The results showed that $0.125 \%$ levobupivacaine and $0.2 \%$ ropivacaine for epidural block by a caudal approach provide similar lumbosacral pain relief, hemodynamic effects, and the degree and the recovery of motor blockade in outpatients with degenerative spinal disease and sciatica.

Key words: Levobupivacaine, ropivacaine, outpatients, epidural block, degenerative spinal disease with sciatica

Pain Physician 2014; 17:525-529 
Epidural injection of local anesthetic agents has been used to treat lumbosacral pain due to degenerative spinal disease and sciatica. Racemic bupivacaine for lumbar epidural block has traditionally been the most widely used in outpatients, but its use has sometimes resulted in fatal cardiotoxicity or neurotoxicity after accidental intravascular injection (1). Levobupivacaine and ropivacaine are associated with less risk for cardiac and central nervous system toxicity and are also less likely to result in unwanted motor blockade in surgical patients. Although epidural levobupivacaine and ropivacaine have been investigated in surgical patients $(2,3)$, there are few reports on the efficacy of epidural block in outpatients with lumbosacral radiculopathy. This study was designed to evaluate the comparative efficacy of epidural block with $0.125 \%$ levobupivacaine and $0.2 \%$ ropivacaine in terms of the sensory and motor blockade and hemodynamic effects in outpatients with degenerative spinal disease and sciatica.

\section{Methods}

With approval of the Institutional Ethics Committee and written informed consent from each patient, we studied 32 patients ( 19 men and 13 women, $26-80$ years old) who had been diagnosed by an orthopedist as having degenerative spinal disease. Plain lumbar $\mathbf{x -}$ rays showed the structural degenerative change, i.e., spur formation, spinal instability, and/or disc narrowing. Magnetic resonance imaging (MRI) showed lumbar herniated interval disc and spinal canal and/or neuroforaminal stenosis. The major symptoms of patients were moderate pain in the low back, buttock, and/or leg regions (Table 1). Patients who had a severe spinal canal stenosis with pseudo-claudication or who had received spinal surgery were excluded from the study. The use of non-steroidal anti-inflammatory drugs (NSAIDs) and steroids had been discontinued one week before the study.

The study was performed in a prospective, randomized, double-blind, and crossover design as follows. Each solution for epidural block, $15 \mathrm{~mL}$ in volume, was prepared by one investigator. A second investigator, who did not know the type of test solution, performed the epidural injection. The variables were measured by a third investigator blinded to the type of test solution. Each patient received all of the test solutions in a randomized order at intervals of one week or more.

Epidural block was performed by a caudal approach in the prone position. After local infiltration with $0.5 \%$ mepivacaine into the skin and subcutaneous tissues, the caudal epidural space was identified by the loss of resistance to saline using a 20-gauge Tuohy epidural needle. The tip of the needle was positioned into sacral canal at the depth of $5.0-6.0 \mathrm{~cm}$ through the sacrococcygeal ligament from the sacral hiatus. After a single injection of $15 \mathrm{~mL}$ of $0.125 \%$ levobupivacaine or $0.2 \%$ ropivacaine was administrated slowly, the patients were immediately placed in the horizontal supine position.

Analgesia was examined bilaterally by the pinprick method with a blunt 27-gauge needle, applied on the mid-clavicular line in both cranial and caudal directions at 15,30,60, and 90 minutes after the end of epidural injection. Motor blockade of the lower limb was evaluated by a modified Bromage scale (grade $0-3$ ) immediately after the assessment of the analgesia level. When the modified Bromage scale had returned to grade 0 , the patient was carefully mobilized to stand up on his or her toes and heals and lastly to slowly walk. Time to micturition was checked and catheterization was performed if necessary. The recovery times from an epidural injection to mobilization, ambulation, and spontaneous micturition were recorded. Pain score was assessed by a visual analogue scale (VAS) before and after epidural blockade. A $100 \mathrm{~mm}$ horizontal VAS with end descriptors of "no pain" and "pain as bad as it could be" was used. Arterial blood pressure (ABP) and heart rate (HR) were recorded throughout the time course.

The Mann-Whitney rank sum test was used for evaluation of the difference between the 2 trials. Wilcoxon's singed rank test was applied on paired data. A value of $P<0.05$ was considered significant.

\section{Results}

There was no incidence of acute intoxication of local anesthetic due to intravascular injection or subarachnoid injection. Three patients were excluded because of lumbosacral pain relief after the first epidural trial or 2 unsuccessful epidural blocks. A total of 29 patients who completed the protocol were included in this study.

There was no significant difference in the upper level of analgesia between the 2 trials: for $0.125 \%$ levobupivacaine trial the median value was L2 (range Th8 - L5) and for $0.2 \%$ ropivacaine trial the median value was L1 (range Th5 - L5). Epidural blockade significantly reduced VAS in each trial $(P<0.05)$, and there was no significant difference in pre- and post-VAS between the 2 trials (Fig. 1). 
Table 1. Clinical details of patients investigated in this study.

\begin{tabular}{|c|c|c|c|c|}
\hline Age & Sex & Diagnosis & Confirmation & Symptoms \\
\hline 42 & M & Spinal stenosis ( radiculopathy ) & Plain X-rays, MRI & Low back pain, leg pain \\
\hline 36 & $\mathrm{~F}$ & Lumbar disc hernia & Plain X-rays, MRI & Low back pain, buttock pain, leg pain \\
\hline 39 & $\mathrm{~F}$ & Lumbar disc hernia & Plain X-rays, MRI & Low back pain, buttock pain \\
\hline 52 & M & Lumbar disc hernia & Plain X-rays, MRI & Low back pain \\
\hline 64 & $\mathrm{~F}$ & Spinal stenosis ( radiculopathy ) & Plain X-rays, MRI & Low back pain, leg pain \\
\hline 69 & $\mathrm{~F}$ & Spinal stenosis ( radiculopathy ) & Plain X-rays, MRI & Low back pain, buttock pain, leg pain \\
\hline 65 & $\mathrm{~F}$ & Spondylolisthesis ( radiculopathy) & Plain X-rays, MRI & Leg pain \\
\hline 68 & $\mathrm{~F}$ & Spinal stenosis ( radiculopathy ) & Plain X-rays, MRI & Low back pain, leg pain \\
\hline 70 & M & Spinal stenosis ( radiculopathy ) & Plain X-rays, MRI & Leg pain \\
\hline 63 & M & Lumbar disc hernia & Plain X-rays, MRI & Low back pain, buttock pain, leg pain \\
\hline 26 & M & Lumbar disc hernia & Plain X-rays, MRI & Low back pain, leg pain \\
\hline 70 & M & Spinal stenosis ( radiculopathy ) & Plain X-rays, MRI & Leg pain \\
\hline 70 & $\mathrm{~F}$ & Spinal stenosis ( radiculopathy ) & Plain X-rays, MRI & Low back pain, leg pain \\
\hline 72 & M & Spinal stenosis ( radiculopathy ) & Plain X-rays, MRI & Low back pain, buttock pain, leg pain \\
\hline $71^{*}$ & M & Spinal stenosis ( radiculopathy ) & Plain X-rays, MRI & Low back pain, leg pain \\
\hline $71^{*}$ & $\mathrm{M}$ & Spondylolisthesis ( radiculopathy ) & Plain X-rays, MRI & Low back pain, leg pain \\
\hline 67 & $\mathrm{~F}$ & Lumbar disc hernia & Plain X-rays, MRI & Low back pain, buttock pain, leg pain \\
\hline 56 & $\mathrm{M}$ & Lumbar disc hernia & Plain X-rays, MRI & Leg pain \\
\hline 49 & M & Spinal stenosis ( radiculopathy ) & Plain X-rays, MRI & Low back pain \\
\hline 45 & M & Lumbar disc hernia & Plain X-rays, MRI & Low back pain \\
\hline 63 & M & Lumbar disc hernia, Spinal stenosis & Plain X-rays, MRI & Low back pain, buttock pain, leg pain \\
\hline 44 & $M$ & Spinal stenosis ( radiculopathy ) & Plain X-rays, MRI & Low back pain, leg pain \\
\hline 64 & $\mathrm{~F}$ & Spinal stenosis ( radiculopathy ) & Plain X-rays, MRI & Low back pain, buttock pain, leg pain \\
\hline 79 & $\mathrm{~F}$ & Lumbar disc disease ( radiculopathy ) & Plain X-rays, MRI & Low back pain, buttock pain, leg pain \\
\hline 75 & M & Spinal stenosis ( radiculopathy ) & Plain X-rays, MRI & Low back pain, leg pain \\
\hline 80 & $\mathrm{M}$ & Spinal stenosis ( radiculopathy ) & Plain X-rays, MRI & Buttock pain, leg pain \\
\hline 83 & $\mathrm{~F}$ & Spinal stenosis ( radiculopathy ) & Plain X-rays, MR I & Low back pain, buttock pain, leg pain \\
\hline 78 & $\mathrm{~F}$ & Spinal stenosis ( radiculopathy ) & Plain X-rays, MRI & Low back pain, buttock pain, leg pain \\
\hline 80 & M & Spinal stenosis ( radiculopathy ) & Plain X-rays, MRI & Leg pain \\
\hline 79 & $\mathrm{~F}$ & Spinal stenosis ( radiculopathy ) & Plain X-rays, MRI & Low back pain, buttock pain, leg pain \\
\hline 84 & M & Spinal stenosis ( radiculopathy ) & Plain X-rays, MRI & Buttock pain, leg pain \\
\hline $59^{*}$ & $\mathrm{M}$ & Spinal stenosis ( radiculopathy ) & Plain X-rays, MRI & Buttock pain, leg pain \\
\hline
\end{tabular}

${ }^{\star}$ Three patients were excluded from the analysis because of pain relief and unsuccessful epidural blocks.

In regards motor blockade, there was no significant difference in the grades of Bromage scale between the 2 trials in any point (Table 2). No patient in either trial developed grade 3 motor block (Table 2).

There were no significant differences in the recovery time to mobilization, ambulation, and spontaneous micturition (Table 3). None of the patients had the prolongation of the recovery time to mobilization, ambulation, and spontaneous micturition and no patients were catheterized during the study for bladder distention in the 2 trials.

There was no significant difference in the ABP (Fig. 2) and HR between the 2 trials throughout the time course.

\section{Discussion}

The results of this study showed that $0.125 \%$ levobupivacaine and $0.2 \%$ ropivacaine for epidural block 


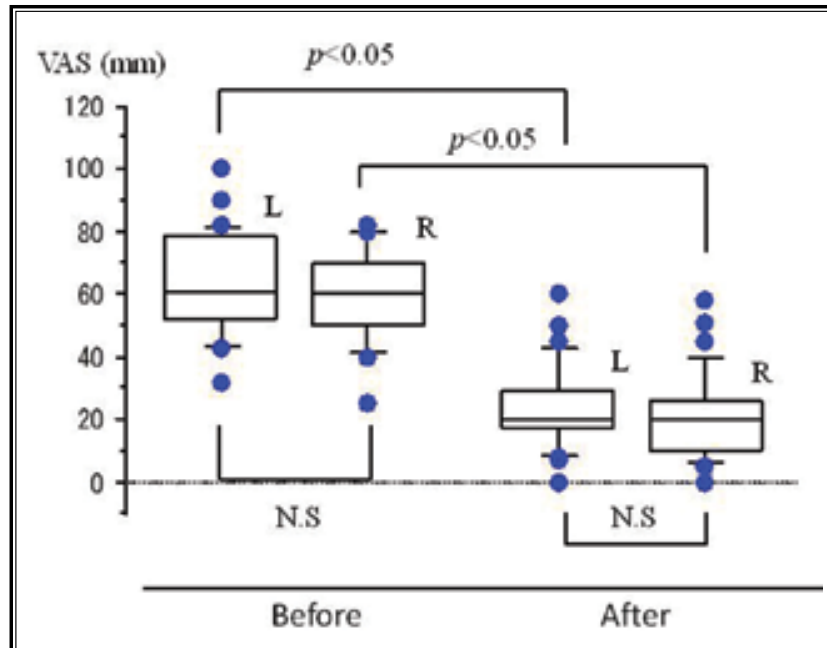

Fig. 1. VAS before and after epidural block with $0.125 \%$ levobupivicaine $(L)$ and $0.2 \%$ ropivacaine $(R)$ (median \pm upper and lower quartiles) VAS =Visual Analogue Scale. After epidural block with 2 trials, VAS showed a significant decrease. There were no significant differences in VAS between the 2 trials.

Table 2. Bromage scale during epidural blockade with $0.125 \%$ levobupivacaine and $0.2 \%$ ropivacaine (mean \pm S.D.).

\begin{tabular}{||c|c|c|c|}
\hline & $\begin{array}{c}\mathbf{0 . 2 \%} \\
\text { ropivacaine }\end{array}$ & $\begin{array}{c}\mathbf{0 . 1 2 5 \%} \\
\text { levobupivacaine }\end{array}$ & P-value \\
\hline Bromage scale 15min & $0.03 \pm 0.18$ & $0.00 \pm 0.00$ & 0.82 \\
\hline Bromage scale 30min & $0.14 \pm 0.35$ & $0.10 \pm 0.40$ & 0.69 \\
\hline Bromage scale 60min & $0.24 \pm 0.44$ & $0.14 \pm 0.44$ & 0.42 \\
\hline Bromage scale 90min & $0.14 \pm 0.35$ & $0.07 \pm 0.37$ & 0.53 \\
\hline
\end{tabular}

Bromage scale: Grade $0=$ no weakness; Grade $1=$ inability to raise extended leg; Grade 2 = inability to flex knee; Grade 3 = inability to move any joint in legs.

Table 3. The recovery times from epidural injection to mobilization, ambulation, and spontaneous micturition (mean $\pm S D$ ).

\begin{tabular}{|c|c|c|c|}
\hline & $\begin{array}{c}\mathbf{0 . 2 \%} \\
\text { ropivacaine }\end{array}$ & $\begin{array}{c}\mathbf{0 . 1 2 5 \%} \\
\text { levobupivacaine }\end{array}$ & P-value \\
\hline Mobilization (min) & $107 \pm 28$ & $102 \pm 48$ & 0.18 \\
\hline Ambulation (min) & $107 \pm 28$ & $103 \pm 48$ & 0.25 \\
\hline $\begin{array}{c}\text { Spontaneous } \\
\text { micturition (min) }\end{array}$ & $132 \pm 79$ & $108 \pm 53$ & 0.15 \\
\hline
\end{tabular}

by a caudal approach provided similar lumbosacral pain relief, the degree and the recovery of motor blockade, and hemodynamic effects in outpatients with degenerative spinal disease and sciatica.

Levobupivacaine, the pure S(-) enantiomer of racemic bupivacaine and ropivacaine, the pure $S(-)$ enantiomer solution are associated with less risk for cardiac and central nervous system toxicity and are also less likely to result in unwanted postoperative motor blockade than racemic bupivacaine (4-7). The potency ratio between bupivacaine, levobupivacaine, and ropivacaine is controversial. Experiments by Kanai et al (8) using anesthetized rats receiving the epidural levobupivacaine and ropivacaine showed that low concentration $(0.25 \%$ and $0.5 \%$ ) levobupivacaine provided more sensory block with the similar motor blockade than ropivacaine. Recent studies seem to indicate that levobupivacaine is slightly more potent than ropivacaine (9); however, other investigations have been shown equipotency $(10,11)$. De Cosmo et al $(2)$ concluded that equivalent volumes of ropivacaine $(0.2 \% \mathrm{w} / \mathrm{v})$ and levobupivacaine $(0.125 \% \mathrm{w} / \mathrm{v})$ provided similar static and dynamic analgesia with similar incidence of minor side-effects after thoracotomy.

The mechanisms of the low back and leg pain in lumbosacral degenerative disease are hypothesized to be ischemic change of nerve roots and activation of neurochemical inflammatory mediators (12). Epidural blocks with local anesthetics could increase the blood flow of the nerve root due by blocking sympathetic nerve fibers, resulting in washing out the inflammatory substances (13). In this study, lumbosacral radicular pain was due to the fifth lumbar nerve or the first sacral nerve in the lesion of the nerve root, and thus a caudal approach for lumbar epidural blockade was used for obtaining the therapeutic effect and for avoiding accidental subdural or subarachnoid blockade.

A faster recovery from motor blockade is required as well as persisting sensory blockade in the therapeutic epidural block for outpatients. In this study, $0.125 \%$ levobupivacaine provided lumbosacral pain relief, sympathetic nerve block, and motor blockade similar to $0.2 \%$ ropivacaine. The upper level of sensory blockade was also similar in all patients. It seems that the epidural solution could spread to the cranial space without severe spinal canal stenosis and reach the injured nerve roots and surroundings. 
Recent studies show that levobupivacaine has similar potency to bupivacaine. Sensory block tended to be longer with levobupivacaine than bupivacaine, amounting to a difference of 23 to 45 minutes with epidural administration (4). With epidural administration, levobupivacaine produced less prolonged motor block than sensory block. In this study, none of the patients showed the prolongation of motor blockade. Therapeutic epidural block with $0.125 \%$ levobupivacaine would be safe without hemodynamic changes in outpatients.

\section{Conclusion}

We concluded that $0.125 \%$ levobupivacaine and $0.2 \%$ ropivacaine for epidural block by caudal approach provided similar lumbosacral pain relief, degree and recovery of motor blockade, and hemodynamic effects in outpatients with degenerative spinal disease.

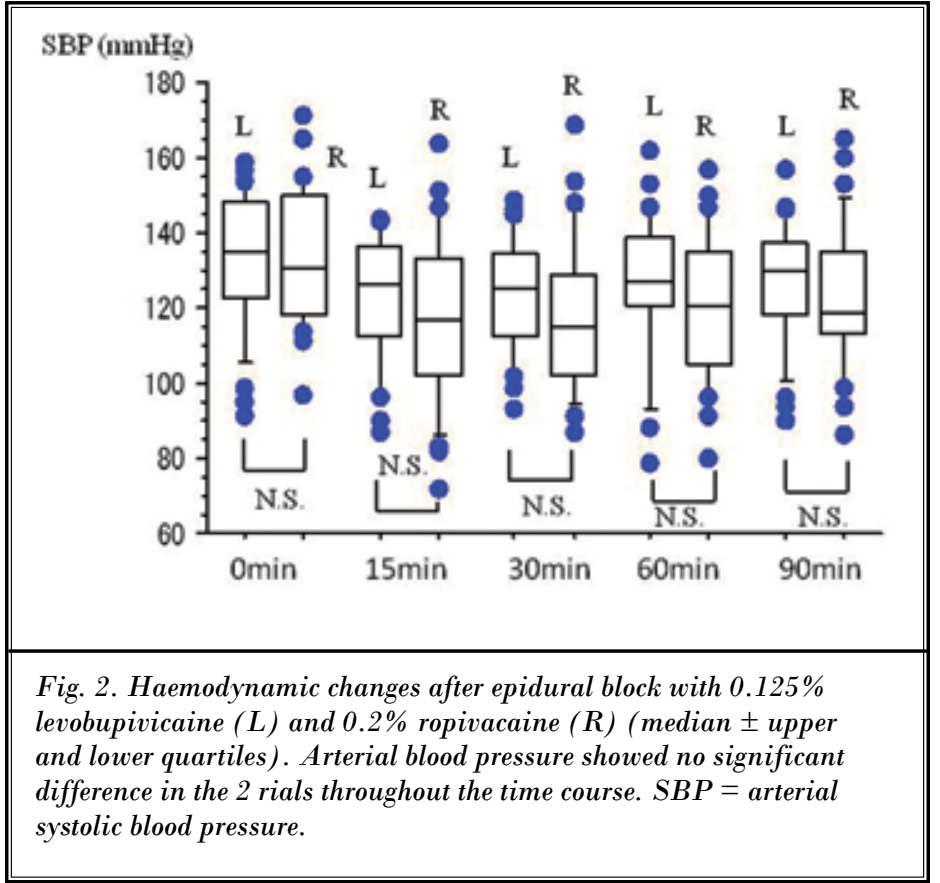

ropivacaine and levobupivacaine. Acta Biomed 2008; 79:92-105

6. Morrison SG, Domingues JJ, Franscarolo P. A comparison of the electrocardiographic cardiotoxic effect of racemic bupivacaine, levobupivacaine, and ropivacaine in anesthetized swine. Anesth Analg 2000; 9:1308-1314.

7. Knudsen K, Beckman SM, Blomberg S. Central nervous and cardiovascular effects of i.v. infusions of ropivacaine, bupivacaine and placebo in volunteers, $\mathrm{Br}$ ] Anaesth 1997; 78:507-514.

8. Kanai Y, Tateyama S, Nakamura T, Kasaba T, Takasaki M. Effects of levobupivacaine, bupivacaine and ropivacaine on tail-flick response and motor function in rats following epidural or intrathecal administration. Reg Anesth Pain Med 1999; 24:444-452.

9. Camorica M, Capogma G, Lyons G, Columb M.; Epidural test dose with levobupivacaine and ropivacaine: determination of ED (50) motor block after spinal administration. Br J Anaesth 2004;
92:850-853.

10. Dubois J, Dreelinck R, Devlieger R, Teunkens A, Vandermeersch E, Van de Velde M. Determination of the full dose-response relation of intrathecal bupivacaine, levobupivacaine, and ropivacaine, combined with sufentanil, for labor analgesia. Anesthesiology 2007; 106:149-156.

11. Andrea C, Roberta S, Giorgio A, Flavio R, Guido F, Marco B, Gianfranco F, Giogio T. Intraoperative epidural anesthesia and postoperative analgesia with levobupivacaine for major orthopedic surgery: A double-blind, randomized comparison of racemic bupivacaine and ropivacaine. J Clinl Anesth 2003; 15:126-131.

12. Olmarker K, Rydevik B. Pathophysiology of sciatica. Orthop Clin North Am 1991; 22:223-234.

13. Yabuki S, Kikuchi S. Nerve root in filtration and sympathetic nerve block. An experimental study of intraradicular blood flow. Spine 1995; 20:901-906. 
